\title{
XXVIII. On the action of a Wehnelt cathode
}

\section{Frank Horton Sc.D.}

To cite this article: Frank Horton Sc.D. (1914) XXVIII. On the action of a Wehnelt cathode , Philosophical Magazine, 28:164, 244-252, DOI: 10.1080/14786440808635204

To link to this article: http://dx.doi.org/10.1080/14786440808635204

\section{冓 Published online: 08 Apr 2009.}

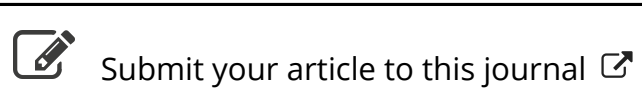

\section{Џll Article views: 5}

Q View related articles

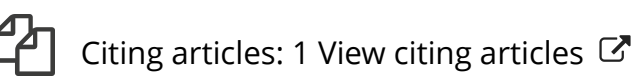


In this formula $\mathrm{X}_{1}$ is expressed in kilovolts per centimetre. If $f$ is the measure of the force in electrostatic units,

$$
f \sqrt{a}=60 \text {. }
$$

Now Zeleny, working with liquid points, obtained the empirical result *

$$
f \sqrt{a}=56 \cdot 9 \text {. }
$$

The similarity of these two results both in form and magnitude of the constant is very striking, especially when the extent of the approximations is considered.

In conclusion, I should like to express my thanks to Professor Townsend both for suggesting the research and for kind help in carrying it out.

XXVIII. On the Action of a Wehnelt Cathode. By Frank Horton, Sc.D. $\dagger$

GINCE the discovery by Wehnelt of the large electron $D$ emission which takes place when a lime-coated cathode is heated in a vacuum, several theories have been put forward to explain its action. One of the earliest of these, and one which received the support of the discoverer of the effect $f$, was that the electrons proceed, not from the glowing oxide, but from the platinum strip or wire upon which it is heated, and that the action of the lime is confined to a reduction of the amount of energy required to liberate the electrons from the metal. More recently the view has been put forward by Fredenhagen $\S$ that the electron emission from glowing lime oncurs as a result of the recombination of calcium and oxygen which have been separated by electrolysis. A modification of this theory has been suggested by Gehrts \|, who assumes that the calcium and oxygen are separated, not by electrolysis, but by thermal dissociation of the lime at the high temperature. This view simplifies the explanation of the manner in which the activity begins. This is supposed by Fredenhagen to be due to the small thermionic current from the platinum support electrolysing the lime in passing through it, and thus giving a supply of calcium and oxygen to start the greater emission from the lime itself.

* J. Zeleny, Phys. Rev. n. s. iii. p. 88, Feb. 1914.

+ Communicated by the Author.

† See Wehnelt, Phil. Mag. [6] x. p. 80 (1905).

$\$$ K. Fredenhagen, Ber. d. Kggl. Sachs. Ges. d. Wiss. lxv. p. 42 (1913).

i) A. Gehrts, Ber. d. Deutsch. Phys. Ges. p. 1047 (1913). 
Fredenhagen's theory has been tested by experiments with Nernst filaments, which give a convenient form of oxide cathode without the complication of a metallic support; and a paper has recently been communicated to the Royal Society in which it is shown that the emission from such an oxide cathode is quite independent of the possibility of electrolysis, and that the emission from the material of the filament at a given temperature is the same whether it is heated in the usual manner by conducting an electric current, or is powdered up and heated upon a platinum strip. Further experiments have been made to test these theories of the origin of the activity of a Wehnelt cathode, and two of these experiments. are described in the present paper. The first was made to test whether the emission from lime depends upon the nature of the material upon which it is heated; the second experiment is a test of the separation of calcium and oxygen by the passage of a thermionic discharge from lime, and of the connexion between the recombination of these elements and the electron emission.

The discharge-tube used in these experiments and the method of heating the Nernst filament and of covering it with lime are described in an earlier paper*. The temperatures were measured by means of a Féry optical pyrometer, for the use of which I am indebted to Professor 'T'. Mather, of the City and Guilds College, London.

\section{A comparison of the Electron emission from Lime heated upon a Nernst flament with that from Lime heated upon Platinum.}

In a recent paper * the author has described an experiment which shows that a large electronic emission is obtained from lime when heated upon a Nernst filament, but attempts to compare the emission with that given by an ordinary Wehnelt. cathode were unsuccessful on account of the difficulty of maintaining the filament glowing in a vacuum at a temperature low enough to enable measurements to be made in the absence of luminous pencils of cathode rays. The appearance of these luminous rays is always accompanied by great overbeating at the points of lime from which they start; so that it is impossible to ascertain to what temperature of the cathode the measured current corresponds.

At temperatures rather lower than that at which these luminous rays are seen, a much fainter luminosity occurs in the discharge-tube and, at the low pressures used in these

* Proc. Camb. Phil. Soc. xvii. p. 414 (1914). 
experiments, seems to fill the whole bulb. This luminosity appears quite gradually as the temperature of the cathode is slowly raised, so that it is very difficult to say when it first begins. It is not accompanied by any sudden increase in the thermionic emission, showing that the increase of temperature of the cathode produced by the luminous discharge is general, and is not confined to a few points of its surface. Further experience of working with Nernst filaments has made it possible to control the heating of a filament even at temperatures as low as $1100^{\circ} \mathrm{C}$., and a comparison of the electron emission from lime heated upon a Nernst filament with that from lime heated upon platinum has now been made. The filament used was covered with pure lime, prepared from marble, in the manner described in the paper already referred to. It was heated by an alternating current, and the thermionic emission was measured in a vacuum obtained by the use of charcoal cooled in liquid air. During the observations there was a faintly luminous discharge through the residual gas; but at the lowest temperatures the luminosity was very difficult to discern, and perhaps was sometimes absent.

For measuring the emission from lime heated upon platinum a fine platinum tube was fitted into the apparatus in the place of the Nernst filament. This tube was covered with lime and could be heated by an alternating current from a transformer. The gas pressure was reduced as low as possible by means of charcoal cooled in liquid air, and the thermionic current was measured as the temperature of the lime-covered tube was gradually raised. A large negative emission occurred immediately the cathode became luminous. The current soon became steady; there was no gradual increase as had been observed in an earlier research with a platinum tube covered with the material of a Nernst filament. and the first observations of the emission at different tensporatures were the largest values obtained.

In comparing the emission from this lime-covered platinum with that from a similarly covered Nernst filament, a difficulty arises on account of the unsaturated nature of the thermionic current given by a lime cathode. Even at the very low pressures and comparatively low temperatures used in these experiments, any increase in the applied E.M.F. caused an increase in the observed emission, and with the highest voltages it was deemed safe to apply, there was no sign of saturation. Since a saturation current cannot be measured, it is necessary, in order to compare the electron emissions in the two cases, to measure the thermionic currents with the 
same potential differences between the electrodes. The difficulty is to allow for the effect of the field due to the heating current. In the case of the Nernst filament there was an alternating potential difference of about 50 volts between the two ends of the cathode, while with the platinum tube about 16 volts were used-the exact value in each case being different at different temperatures. It was finally decided to apply between the terminals of the discharge-tube a potential difference of 207 volts in the case of the platinum tube, and 168 volts in the case of the Nernst filament-these being values easily obtained from the high-potential battery, while the difference between them makes allowance for the difference in the field due to the heating current in the two cases. For both cathodes curves connecting the thermionic current and the temperature were drawn, and from these curves the following values of the emissions at different temperatures are taken. The area of the cathode covered by lime was approximately the same in the two cases. The numbers given in the table are the largest values of the

\begin{tabular}{|c||c|c|}
\hline $\begin{array}{c}\text { Temperature, } \\
\text { centigrade. }\end{array}$ & \multicolumn{2}{|c|}{ Thermionic Current in $10^{-5}$ ampere. } \\
\cline { 2 - 3 } & Lime on Filament. & Lime on Platinum. \\
\hline 1100 & $12 \cdot 9$ & $4 \cdot 1$ \\
1150 & $20 \cdot 2$ & $10 \cdot 2$ \\
1200 & $34 \cdot 0$ & $23 \cdot 0$ \\
1230 & $46 \cdot 2$ & $33 \cdot 9$ \\
\hline
\end{tabular}

thermionic current in both cases. On continued heating, the emission from the lime-covered filament decreased more rapidly than that from the lime-covered platinum, apparently because the lime adhered more readily to the metal than to the material of the filament. From both lime cathodes there was a continued slight emission of gas which, if it were very unequal in the two cases, would render a comparison of the emissions impossible; for, with the strong electric fields used, ionization by collisions would play an important part in deciding the value of the measured thermionic current. Throughout the experiments a carbon tube cooled in liquid air was in connexion with the discharge-tube so as to keep the pressure low, and as nearly constant as possible.

From the table it will be seen that the numbers measuring the electron emission from lime heated upon a Nernst 
filament are rather larger than those for the case of lime heated upon platinum; but considering the difficulties in making the comparison the differences are small, and we are justified in concluding that, in these two cases, the emission from lime does not depend on the nature of the material upon which it is heated.

\section{7 he Liberation of Gas from a Wehnelt Cathode.}

Observations have been made of the rate at which the gas pressure in the apparatus increases when the lime-covered platinum tube is acting as a cathode, and when it is heated but no thermionic current is allowed to pass. For this purpose the gas pressure was taken down as low as possible by means of a carbon tube cooled in liquid air; this tube was then shut off by a tap. The mercury-pump was separated from the discharge-tube so that the volume into which the evolved gas could diffuse was considerably reduced, being now only that of the discharge-tube, the phosphorus pentoxide drying-tube, the McLeod gauge, and the connecting tubes-in all 550 c.c. Taking observations at intervals of 15 minutes with the platinum tube at $1360^{\circ} \mathrm{C}$., alternately with no thermionic current passing and with a current of an average value of 8 milliamperes, it was found that the pressure continually increased during the first two hours' heating, but at a rather greater rate when the discharge was passing than when no discharge passed. The increase of pressure per minute, however, in both cases became less as the heating was continued, until after two hours there was a small decrease of pressure in the absence of the discharge. The largest observed increase of pressure in 15 minutes with a thermionic current of 8 milliamperes was $\cdot 0007 \mathrm{~mm}$., the mean increase during the periods with no discharge immediately preceding and following this being $0002 \mathrm{~mm}$. If the difference between these two increases of pressure is due to oxygen gas liberated by electrolysis of the lime under the action of the thermionic current passing through it, the amount of oxyger: so liberated is less than one-thousandth of that which would be set free according to Faraday's law, on the assumption that the conduction of the lime is entirely electrolytic, and that no recombination of the separated elements takes place. It was thought possible that part of the difference between these two increases of pressure was due to the extra heating of the residual gas by the passage of the luminous discharge. Observations were therefore made to test this; but it was found that the pressure alteration due 
to the heating of the gas by the glowing cathode itself was not more than one-tenth of the increase observed above, and was hardly measurable.

After the lime-covered platinum tube had been heated for some hours it was always found that the passage of the luminous discharge was accompanied by a liberation of gas, and that subsequent heating with no thermionic current passing decreased the pressure; the actual numbers were not very regular, but the same general result was always obtained.

The most obvious explanation of this result is that oxygen is liberated by electrolysis of the lime, and that recombination of oxygen and calcium goes on when the cathode is afterwards warmed in the liberated gas. Assuming that this is the correct explanation, it follows that recombination is also going on at the sanie time as the electrolysis, and the question arises: Is this recombination the cause of the electron emission? To test this point observations of the pressure were taken at intervals of 15 minutes with the high potential continually applied to the terminals of the discharge-tube. During the first 15 minutes a temperature of about $1400^{\circ} \mathrm{C}$. was maintained, and a thermionic current of about 4 milliamperes passed. The temperature was then lowered to about $600^{\circ} \mathrm{C}$., and kept at that value for 15 minutes, after which it was raised again to $1400^{\circ} \mathrm{C}$, several observations at these two temperatures being taken alternately. With the cathode at the lower temperature, no thermionic current could be detected with a galvanometer giving $\mathbf{l}$ division deflexion for a current of $1.94 \times 10^{-9}$ ampere; but there was an average decrease of pressure of $\cdot 00063 \mathrm{~mm}$. during these periods of 15 minutes. At the higher temperature, with the thermionic current passing, there was an average increase of pressure of about the same magnitude. Since the effect of the decreased temperature of the cathode upon the gas pressure is almost inappreciable, it is evident that there was an absorption of gas taking place while the cathode was heated at $600^{\circ} \mathrm{C}$. This absorption is presumably due to the union of electrolytically liberated oxygen and calcium, and it was not accompanied by any detectable electron emission. It follows, therefore, that the electron emission observed at the higher temperature cannot be due simply to the recombination of electrolytically separated calcium and oxygen; at all events the existence of the high temperature is an essential condition.

A similar conclusion also follows from the results of some experiments made by the author in 1906 , when comparing 
the electron emission from calcium with that from lime *. In these experiments a platinum strip was covered with calcium by sublimation in a vacuum; an excess of oxygen was let into the discharge-tube and the calcium was oxidized to lime. No detectable ionization occurred during this process of oxidation in the cold, nor even at a temperature of $500^{\circ}$ or $600^{\circ} \mathrm{C}$., under which conditions the oxidation must have been very rapid. It was only when the lime formed had been raised to $700^{\circ} \mathrm{C}$. that a measurable thermionic carrent was obtained.

In this part of the present paper the term "luminous discharge" has so far been used to indicate that faint laminosity of the residual gas which appears gradually as the temperature of the cathode is raised, but which is not accompanied by luminous pencils of cathode rays. At higher temperatures, or with greater applied potential differences, the nature of the discharge alters; brightly luminous pencils of cathode rays appear, starting from points of lime which are visibly hotter than the rest of the cathode, and at the same time there is a sudden increase in the thermionic current. The alterations of pressure which occur during the passage of such a discharge are generally quite different from those recorded above. In the early stages of heating a cathode newly coated with lime there is usually a considerable evolution of gas; but if a brightly luminous discharge is sent from a cathode which has been used for some time, through air at a low pressure, there is nearly always a diminution of pressure produced. This effect was observed by the author some time ago $t$, and a spectroscopic examination was made to see if the passage of the discharge alters the chemical constitution of the residual gas. Some new lines in the red part of the spectrum were observed, but these were shown to be due to mercury $¥$.

During experiments with this type of discharge the current passing is several times as large as that with no luminous pencils of cathode rays; and it is certainly remarkable that a thermionic current of 4 milliamperes is accompanied by an increase of gas pressure in the apparatus, whereas a diminution of pressure is produced by a current of 20 milliamperes. The effect of these large currents is, however, uncertain; on some occasions, even after long-continued heating, an increase of pressure was produced. The diminution of pressure when

* Phil. Trans. A. ecrii. p. 149 (1907).

† Phil. Mag. $[6]$ xi. p. 505 (1006).

¥ Proc. Camb. Pliil. Soc. xiv. p. 501 (1908). 
the heavy discharge passes may perhaps be due to the high temperature of the lime at the points from which the cathode rays proceed; and it is interesting to note in this connexion that some time ago $\mathrm{Sir}$ J. J. Thomson* suggested that the origin of the large electron emission from barium oxide was connected with a chemical transformation from $\mathrm{BaO}$ to $\mathrm{BaO}_{2}$ at the high temperature of the cathode. A peroxide of calcium also exists, so that it is possible that a reversible reaction of this nature goes on in the case of lime. It is, however, very difficult to obtain accurate information about chemical reactions which may be taking place at the high temperatures and low pressures of these experiments.

\section{Conclusion.}

From experiments made by the author in 1906 it appeared that the activity of a Wehnelt cathode is not due to an escape of electrons from the molecules of lime simply as a result of an increase in their thermal energy; for on this view we should expect that the presence of the electronegative oxygen atom in the lime molecule would hinder the escape of electrons, and that, in consequence, the electron emission from lime would be less, at a given temperature, than the emission from the same amount of calcium in the metallic state. The experiments referred to showed that the reverse was the case, and that the emission from calcium is less than that from lime. The results of the experiments described in the present paper may be summarized as follows :-

(1) The electron emission from a Wehnelt cathode has its origin in the lime itself, and the lime does not merely serve to help the electrons to escape from the metal.

(2) When an electric current passes through lime at a bigh temperature, the amount of oxygen liberated is only a very small fraction of what would be expected if the conductivity of the lime were entirely electrolytic. It has been suggested that the conductivity is entirely electrolytic, and that the products of electrolysis diffuse through the lime and recombine; but it appears to the author to be improbable that such recombination should go on, so rapidly and completely, through a lajer of solid lime, especially as the charged oxygen atoms would be liberated on the vacuum

* 'Conduction of Electricity through Gases,' p. 427. Camb. Univ. Press, 1906. 
side of the oxide layer, and with a strong electric field tending to drag them away from the cathode.

(3) The chemical combination of calciurn and oxygen does not by itself give rise to any detectable electron emission.

From (2) and (3) it appears that neither the theory of Fredenhagen nor the modification of that theory proposed by Gehrts can be accepted as an explanation of the activity of the Wehnelt cathode.

The Carendish Laboratory, Cambridge.

XXIX. The Possible Dependence of Gravitational Attraction on Chemical Composition, and the Fluctuations of the Moon's Longitude which might result therefrom. By C.V. Binton, D.S.**

1. 70 what degree of accuracy is the Newtonian coefficient of gravitation a universal constant of matter independent of. chemical and physical properties? To decide this question, Newton himself made experiments on a number of substances; and later Bessel $\dagger$, as the result of a lengthy investigation, found that, within the limits of experimental error (one part in 60,000), the weights of bodies were proportional to their masses; the substances examined including brass, iron, zinc, lead, silver, gold, meteoric iron, meteoric stone, marble, clay, and quartz. Bessel, like Newton, used a pendulum method.

2. In modern determinations of the acceleration due to gravity, if observations were repeated at a given station with pendulums of different materials, far greater accuracy could be attained; and additional refinements could no doubt he introduced where the comparative behaviour of different pendulums was the sole subject of research. But experimenters are not readily attracted to laborious tasks from which only null results are expected.

3. The planets of the solar system, differing considerably in mean density amongst themselves, may he supposed to differ correspondingly in composition, so that the exactitude with which their orbits conform to Kepler's third law is reasonably regarded as evidence of the close uniformity of

* Communicated by the Author.

† "Versuche uiber die Kraft mit welcher die Erde Körper ron verschiedener Beschaffenheit anzieht." Beslin Abhandl. 1830, pp. 41-102; Pogg. Ann. xxv. 1832, pp. 401-417, reprinted in Astron. Nachr. x. 1833, col. $97-108$. 